\title{
A Miniature Mobile Robot Developed to be Socially Integrated with Species of Small Fish
}

\author{
Frank Bonnet, Stefan Binder, Marcelo Elias de Oliveria, José Halloy, and Francesco Mondada
}

\begin{abstract}
A robot accepted by animals as conspecific is a very powerful tool in behavioral biology, particularly in studies of gregarious animals. In this paper we present the first results of acceptance of a robotic fish designed for experiments on collective animal behavior. The robot consists of two modules: a fish lure fixed on a magnetic base and a miniature mobile robot guiding the lure from below the experimental tank. In order to study the acceptance of the robot among living fish, we varied several parameters of the system and used design of experiments methods to reduce the number of performed experiments and determined the impact of each factor on the acceptance of the robot among a small group of real fish. While a brief comparison of the mean distance of the fish to the robot tends to indicate that the fish are attracted by the lure, a linear model of the acceptance of the robot is presented. Results of this study can be used to improve the design and control of the robot for further animal-robot interaction experiments.
\end{abstract}

\section{INTRODUCTION}

One of the long-standing interests in behavioral studies is to understand relationships between stimulus and response. In order to study these mechanisms, researchers often use specially designed mock-ups whose aspect and behavior can be controlled to monitor responses of animals under test. In recent years, as technology became more advanced and affordable, robotic devices have been introduced into animal societies to generate those stimuli. One of the first concrete example was the Leurre project, where a mixed society consisting of cockroaches and mobile robots was created [1]. These types of experiments have been extended to more complex species, such as beetles [2], fish [2] or even chicken [3].

The group behavior of the zebrafish Danio Rerio, a fish used in hundreds of laboratories worldwide for different scientific topics [4], already raised the interest of biologists, and several examples of automated lure designed to interact with zebrafish have already appeared. For instance, in [5] and [6], the zebrafish response to a robotic fish with the same ratio size as zebrafish, a beating tail and different colorations was observed. In [7], [8] and [9], a lure attached to a support is moved using a mobile robot outside the aquarium and controlled using a tracking software.

F. Bonnet, M. E. de Oliveria and F. Mondada are with the Robotic Systems Laboratory, School of Engineering, Ecole Polytechnique Fédérale de Lausanne, Lausanne, Switzerland frank. bonnet depfl.ch

S. Binder is with the Transport and Mobility Laboratory, School of Engineering, Ecole Polytechnique Fédérale de Lausanne, Lausanne, Switzerland s.binderdepfl.ch

J. Halloy is with Laboratoire Interdisciplinaire des Energies de Demain, Université Diderot Paris VII, Paris, France jose.halloyeuniv-paris-diderot. fr
In the last couple of years, we have designed and evaluated a robot that can be socially integrated into a zebrafish shoal [10]. The current version of the robot that is used during the experiments presented in this paper can be seen in Fig. 1. The main advantage of our robot when compared to previously published solutions, is its small dimensions that, adding that it is controlled using wireless link, offers the possibility to have several lure able to move next to each other inside an aquarium, for multi-robot experiments. Furthermore, the robot has been conceived so that the lure reaches speeds and accelerations of respectively $0.5 \mathrm{~m} / \mathrm{s}$ and $2 \mathrm{~m} / \mathrm{s}^{2}$, which are typical for zebrafish based on the available experimental results [11] and that have been observed on our own animals. The robot is powered using conductive plates that are installed on the experimental setup and thus the experiments can last several hours. In our studies, we take zebrafish as an example to validate our results, but the system could also apply for other species of small fish.

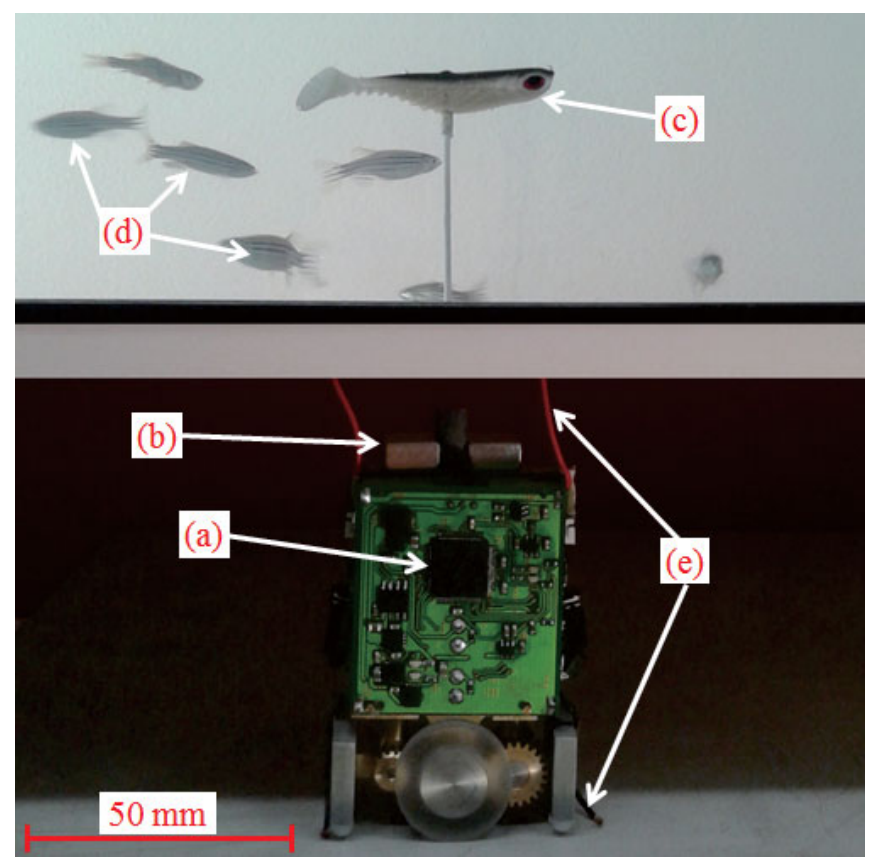

Fig. 1: Current version of the our robotic system for mimicking fish behavior. a) Mobile robot moving below an aquarium. b) Magnets to couple magnetically the mobile robot with the lure module. c) Lure module moving inside the aquarium. d) Living zebrafish swimming in the background. e) Electric brushes to retrieve the power from conductive plates to power the mobile robot. 
In this paper, preliminary results of acceptance of our robotic fish by a small group of living zebrafish are presented. Several parameters were tested: the tangential speed of the robot, the aspect of the lure, the type of trajectory made by the robot and the motion continuity of the lure. We used a Design of Experiment (DOE) analysis in order to optimize the number of experiments and to determine the acceptance of the robot by the zebrafish with respect to the different parameters. These experiments were conducted during the design phase of the robot and their main purpose was to know which configuration of the robot's design and control need to be improved to increase the acceptance of the robot among the fish shoal.

The paper is organized as follows: Section II provides a description of the robot design and the experimental setup, Section III describes the experiments that were made, Section IV shows the application of the method DOE to characterize the impact of the factors on the results, Section V presents the results and the discussion and, finally, Section VI concludes the paper.

\section{HARDWARE AND SOFTWARE}

\section{A. Experimental setup}

The experimental setup that is used for the experiments consists of an aquarium of $1000 \mathrm{~mm} \times 1000 \mathrm{~mm}$ of surface covered on the inside with white teflon sheets (Fig. 2). These sheets are installed in order to avoid reflection on the glass and to have a smooth surface for the motion of the lure module inside the aquarium. The tank is filled with water up to a level of $100 \mathrm{~mm}$. According to [4], this level of water is not introducing more stress for the fish and furthermore, the lure, whose height cannot vary, will be more visible for the fish that are swimming around. The water temperature is set to $26^{\circ} \mathrm{C}$, as suggested by [4]. The robot is moving under the aquarium, and the motion is transmitted to the lure using magnets. The robot is powered using two conductive plates, one glued on the bottom of the aquarium and one on the support on which the robot is moving.

\section{B. Mobile Robot}

The mobile robot has a differential drive configuration, with two independently actuated wheels (Fig. 1). The length of the mobile robot is $43 \mathrm{~mm}$, the width $22 \mathrm{~mm}$ and the height $67 \mathrm{~mm}$. The mass of the mobile robot is 80 grams.

The electronic architecture of the mobile robot is presented in Fig. 3. The power supply is done through electric cables (brushes) that slip against two copper conductive plates situated under the aquarium's floor (positive) and on the support on which the robot is moving (negative). Two SuperCaps of 1 farad each with a dual ideal diode system are used in case the brushes are not in contact with the conductive plates for a couple of seconds in order to have a continuous powering of the robot. Proximity sensors are installed on the front and back of the robot in order to avoid obstacles or the border of the arena in the case when the tracking is not working. A bluetooth device is used for wireless communication to control the robot.

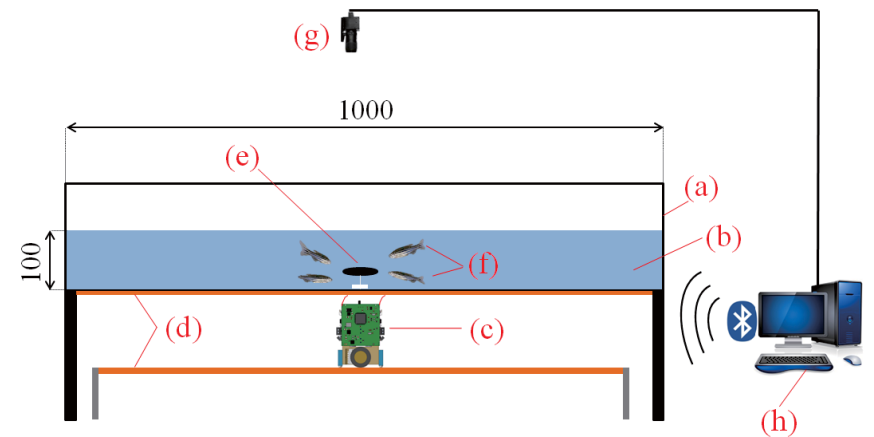

Fig. 2: Experimental setup used during the experiments. a) Aquarium of $1000 \mathrm{~mm}$ x $1000 \mathrm{~mm}$ x $250 \mathrm{~mm}$. b) Water layer of $100 \mathrm{~mm}$. c) Mobile robot moving under the aquarium. d) Conductive plates to power the mobile robot. e) Lure inside the aquarium linked to the mobile robot through magnetic coupling. f) Living zebrafish. g) Camera used to track the lure and the zebrafish. h) A computer that process the camera frames and remotely controlled the robot via Bluetooth protocol.

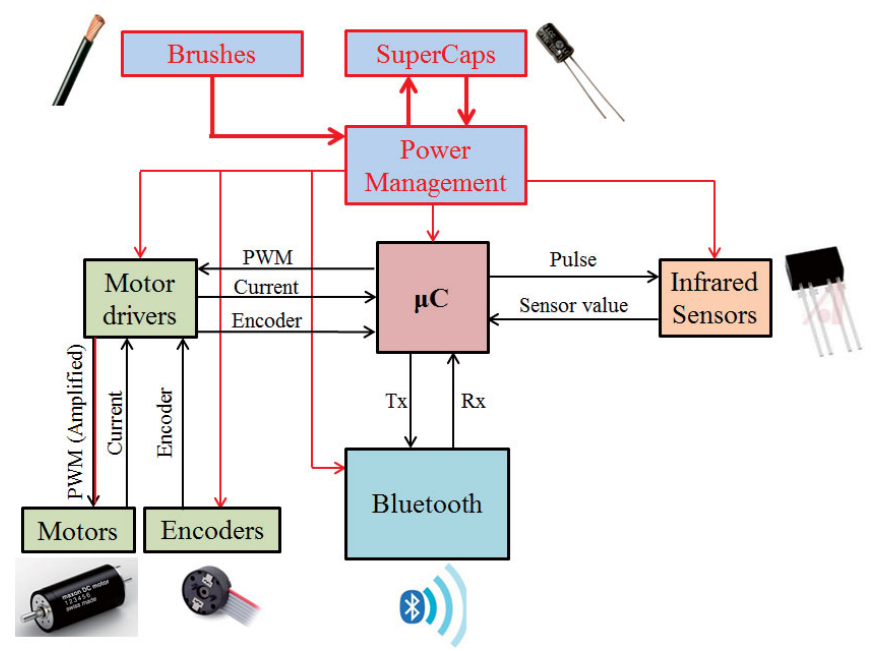

Fig. 3: Electronic architecture of the mobile robot. The microcontroller, a dsPIC33FJ128GP804, is monitoring the sensors and motors, and transmit through the Bluetooth module. The brushes are used to power the system and load the SuperCaps, and when the contact between the brushes and the conductive plates is lost, the SuperCap can furnish the power for the whole system.

On the control side, an ASEBA Virtual Machine (AVM) [12] is running onboard each mobile robot. ASEBA is an event-based architecture for real-time distributed control of robots. Running ASEBA scripts inside each virtual machine help us to reprogram the low-level behavior of each robot without flashing each time their microcontrollers. ASEBA integrated with D-Bus allows access to each robot from high level language such as $\mathrm{C}++$, Python or ROS using a software hub called Medulla [13]. Figure 4 shows the global software architecture of our system. 


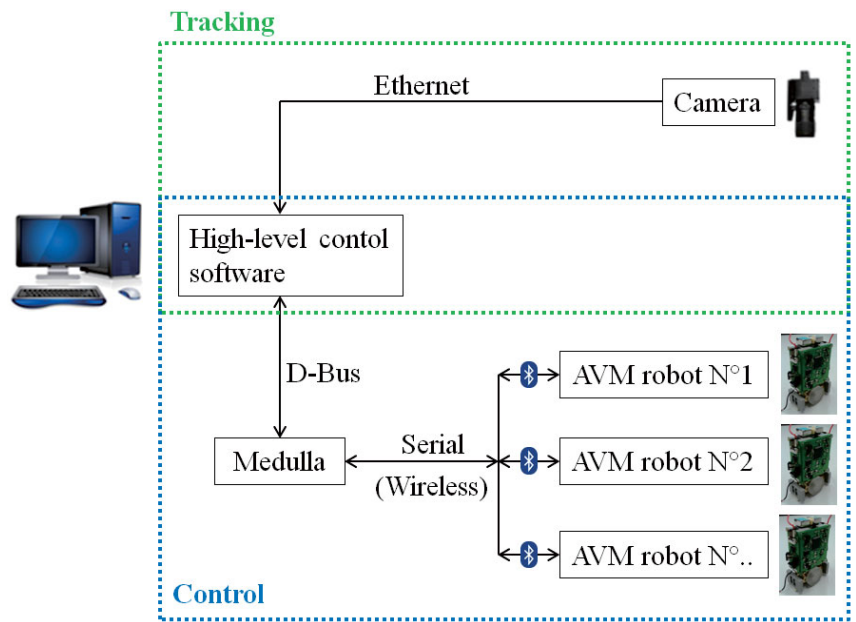

Fig. 4: Control architecture of the mobile robots. The highlevel software is tracking each lures and fishes from frames grabbed by a Camera. The same software is used to control the robot by sending the desired speed of the motor's wheels to each AVM connected through D-Bus using Medulla [13].

\section{Tracking}

During the experiments, a blob detection based algorithm retrieves the pose (position and orientation) of the lure and zebrafish (Fig. 5). A proportional controller is used to move the robot to a target. In order to make the robot follow a trajectory, the target is updated at a certain time step along the trajectory and the lure follows it with a speed proportional to the update time rate of the target.

\section{Lure module}

The lure is moving at a constant water level which will depend on the height of the support. In this case, we chose a support's height of $5 \mathrm{~cm}$ in order to have the lure moving at the middle of the water level. The base of the lure module is painted in white in order to blend into the white background. The lure is fixed on the base and can not rotate.

Two different lures were used (Fig 6). The first one is a rigid black ellipsoid made of Acrylonitrile Butadiene Styrene (ABS) and the second one is a soft fishing lure.

\section{E. Zebrafish}

For the experiments performed, we used 10 zebrafish Danio Rerio, with short fins. These zebrafish were acquired in a pet shop, and are stored in a 60 litres housing aquarium. The average total length of our zebrafish is $\sim 40 \mathrm{~mm}$. The water temperature of the housing aquarium is $26^{\circ} \mathrm{C}$. The fish are fed twice a day using a food distributor with commercial food. The enrichment in the aquarium consists of plastic plants, cladophoras, gravel, rocks and aquatic snails.

\section{DESCRIPTION OF THE EXPERIMENTS}

\section{A. Licence}

The experiments performed in this study were conducted under the authorization $N^{\circ} 2778$ delivered by the Department of Consumer and Veterinary of the Canton de Vaud

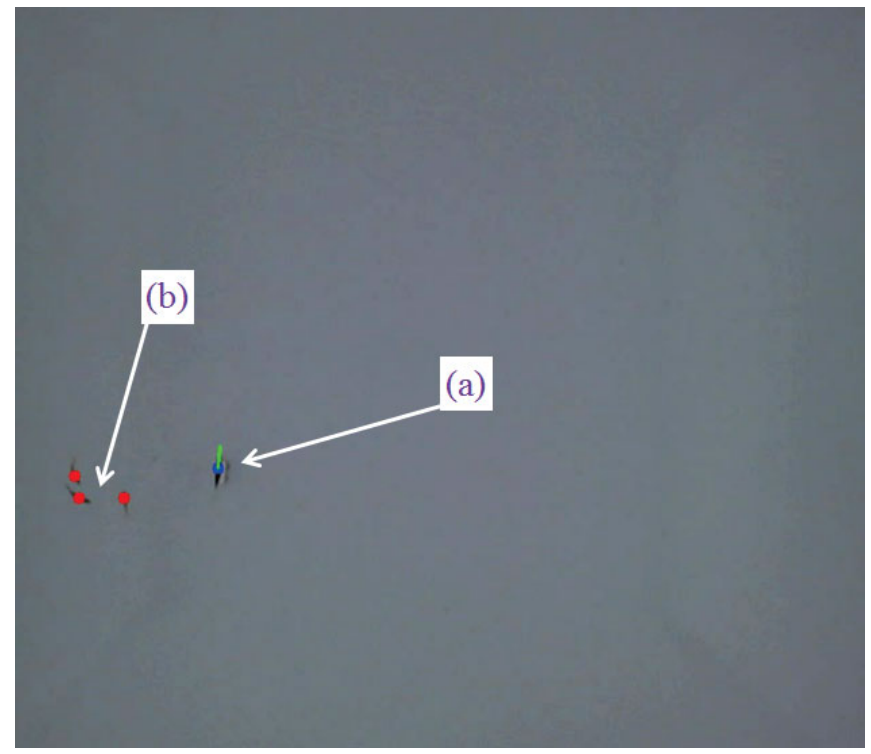

Fig. 5: The result of the blob detection based software used for the tracking and control of the robot. a) Lure inside the tank, the blue dot and the green line indicates the position and orientation respectively. b) Three living zebrafish and the red dots that indicate their positions.

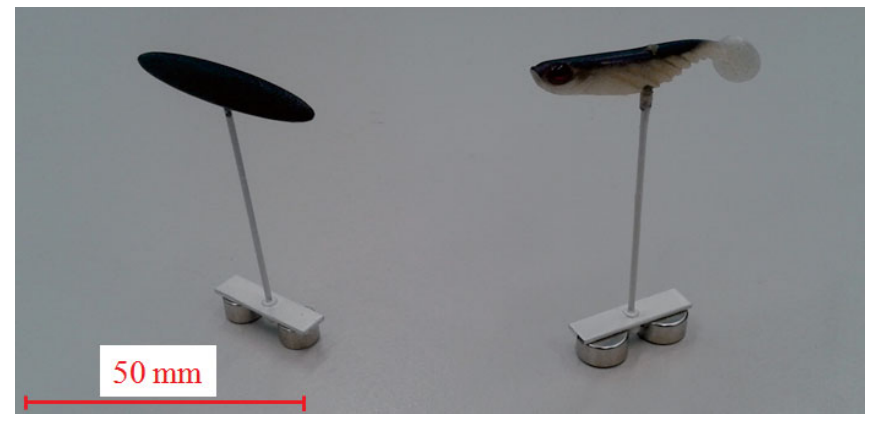

Fig. 6: Lure modules. Left: a rigid black ellipsoid lure. Right: a soft fishing lure. The base is painted in white so it is merged with the white background inside the aquarium.

(Switzerland) after submission to the state ethical board for animal experiments.

\section{B. Manipulation}

For each experiment, three zebrafish out of a shoal of ten individuals were placed inside the experimental tank. The three zebrafish were selected randomly among the entire group in order to reduce learning possibility by the fish from the previous experiments.

Once inside the tank, we let the fish acclimatize during 15 minutes in order to neglect the effect of the stress due to the transfer between the housing aquarium and the experimental tank. Afterwards, we placed the fish replica inside the tank and started the experimentation. The duration of each measurement was 30 minutes, thus the entire manipulation lasted 45 minutes. 


\section{Responses and factors}

In this experiment, four factors were considered to explain the response: see Table I for an overview of the factors.

\begin{tabular}{lccc}
\hline Factor name & Variable & $\begin{array}{c}\text { Min value } \\
(-1)\end{array}$ & $\begin{array}{c}\text { Max value } \\
(+1)\end{array}$ \\
\hline Lure & $X_{1}$ & Fake fish & Ellipsoid \\
Robot's trajectory & $X_{2}$ & (Half-)circles & Circles \\
Robot's movement & $X_{3}$ & Jerks & Continuous \\
Robot's speed [cm/s] & $X_{4}$ & 3 & 6 \\
\hline
\end{tabular}

TABLE I: Factors of the experiment.

The first studied factor was the shape of the lure: it can either be of ellipsoidal shape or represent a fake fish (Fig. $6)$. The idea here is to assess if the aspect of the lure has an influence on the perception of the zebrafish. The second factor describes the different possible trajectories of the robot: it can either turn in circles in the whole aquarium or alternate between full circles and half-circles. Indeed, the zebrafish have a tendency to swim along the border of the tank, as they feel more protected near walls than in the central area of the tank [4]. We wanted to analyse if the robotic fish can influence the group of fish to explore the central area. The robot's movement will be included as a third factor: it can either move continuously or with forward jerks (thus mimicking more closely the fish behaviour). Finally, the robot's speed is a continuous factor, varying between 0.03 and $0.06[\mathrm{~m} / \mathrm{s}]$. This speed range was determined by measuring the speed distribution of the zebrafish.

The responses of the experiment were twofold. The first response analysed was the mean distance between the zebrafish and the lure during the experiments. By measuring this value, one can assess the acceptance of the robot fish in the fish shoal. It is measured in [mm]. The second analysed response is the number of times during the experiment a fish is situated in a radius of $10 \mathrm{~cm}$ around the lure.

The analysis of water flows generated by the two different lures for which fish are very sensitive due to their lateral line were not considered in these experiments, but will be investigated in further studies.

The duration of the experiments was 30 minutes and the fish were randomly selected for each experiment among 10 fish. We considered that measurements on only one trial per experiment were relevant enough for the tests that we have performed.

\section{DESIGN OF EXPERIMENTS}

\section{A. Model description}

A linear model with interactions was selected to analyse the effect of the four different factors on the response of the zebrafish.

The model can be written as follows [14]:

$$
Y=a_{0}+\sum_{i=1}^{n} a_{i} X_{i}+\sum_{i, j=1, j \neq i}^{n} a_{i j} X_{i} X_{j}
$$

where the $X_{i}$ stand for the values of the factors, $a_{i}$ are the model coefficients associated to $X_{i}, Y$ is the response variable and $n$ the number of factor, 4 for the case of this study.

In a full factorial design, a total of $2^{4}=16$ experiments would be needed, as four factors explain the response variable. In order to reduce the number of experiments (which are time- and resource-consuming), a fractional factorial design [15] of type $2_{I V}^{4-1}$ was used. Hence, only $2^{3}=8$ experiments were needed. This reduction in the number of experiments comes at a cost, however, some of the coefficients are now aliased. This design having a resolution of $I V$, neither main effects $a_{i}$ are confounded between each other, nor main effects with first-level interaction terms $a_{i j}$. However, first-level interaction terms $a_{i j}$ are confounded between each other and main effects $a_{i}$ are confounded with second-level interaction coefficients $a_{i j k}$ [16]. If one neglects second-level and higher-level interaction terms, this design is able to determine the main effects without bias [14]. However, first-level interaction terms are aliased: $a_{12}$ with $a_{34}, a_{13}$ with $a_{24}$ and $a_{14}$ with $a_{23}$.

By using the generator $+4=123$, the following matrix of experiments $E$ is obtained:

$$
E=\left[\begin{array}{cccc}
X_{1} & X_{2} & X_{3} & X_{4} \\
1 & 1 & 1 & 1 \\
1 & 1 & -1 & -1 \\
1 & -1 & 1 & -1 \\
1 & -1 & -1 & 1 \\
-1 & 1 & 1 & -1 \\
-1 & 1 & -1 & 1 \\
-1 & -1 & 1 & 1 \\
-1 & -1 & -1 & -1
\end{array}\right]
$$

Hence, the matrix of the model with interactions $X$ is given by

$$
X=\left[\begin{array}{cccccccc}
a_{0} & X_{1} & X_{2} & X_{3} & X_{4} & X_{12} & X_{13} & X_{14} \\
1 & 1 & 1 & 1 & 1 & 1 & 1 & 1 \\
1 & 1 & 1 & -1 & -1 & 1 & -1 & -1 \\
1 & 1 & -1 & 1 & -1 & -1 & 1 & -1 \\
1 & 1 & -1 & -1 & 1 & -1 & -1 & 1 \\
1 & -1 & 1 & 1 & -1 & -1 & -1 & 1 \\
1 & -1 & 1 & -1 & 1 & -1 & 1 & -1 \\
1 & -1 & -1 & 1 & 1 & 1 & -1 & -1 \\
1 & -1 & -1 & -1 & -1 & 1 & 1 & 1
\end{array}\right]
$$

where the first column corresponds to the constant coefficient $a_{0}$ and is a column of ' 1 ', the next three columns correspond to the main effects $a_{1}, a_{2}, a_{3}$ and $a_{4}$ and are composed of the four columns of the matrix of experiments $E$. The last three columns were obtained by multiplying the columns of the matrix of experiments as they correspond to the interacting factors [16].

\section{RESULTS AND DISCUSSION}

Table II shows the results that were obtained from the eight performed experiments. 


\begin{tabular}{lcc}
\hline Run & Mean Distance $(\bar{d}[\mathrm{~mm}])$ & \# of times near robot $(T[-])$ \\
\hline 1 & 414.27 & 274 \\
2 & 354.05 & 2895 \\
3 & 420.90 & 1003 \\
4 & 372.99 & 1706 \\
5 & 401.88 & 1327 \\
6 & 407.87 & 1173 \\
7 & 407.57 & 949 \\
8 & 390.47 & 1325 \\
\hline
\end{tabular}

TABLE II: Results obtained from the 8 experiments performed.

In order to assess if the fish seem attracted by the lure, we have compared the results obtained from the mean distances with an experiment in which only the three zebrafish were swimming, without any lure inside the tank. We have simulated a lure that reproduced the same movements as the robot and computed the distance between each zebrafish and this virtual lure. We have obtained a value of $465.88 \mathrm{~mm}$ for the average distance, which is above all the distances obtained during the experiments (Table II). Thus, we have an indication that the zebrafish seem to be attracted by the lure when the latter is moving inside the tank.

The coefficients of the linear model have been estimated using least squares regression:

$$
\hat{a}=\left(X^{T} X\right)^{-1} X^{T} Y,
$$

where $Y$ is the vector of results. As the factorial design is orthogonal, this equation reduces to

$$
\hat{a}=\frac{1}{N} X^{T} Y
$$

where $N$ is the number of experiments ( $N=8$ in our case).

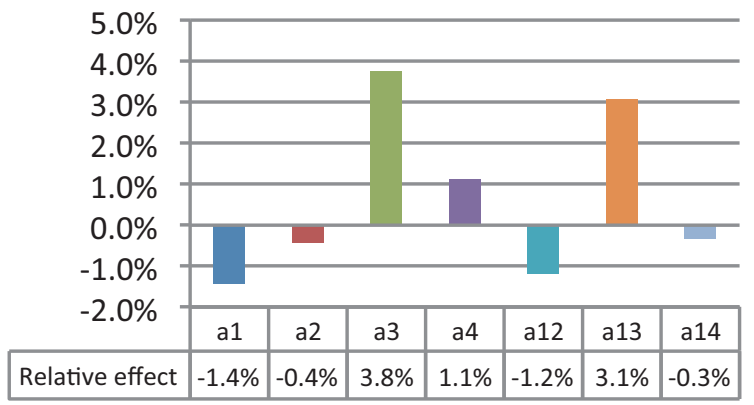

Fig. 7: Relative effects of the factors on the first response, the mean distance between the lure and the zebrafish $(\bar{d})$.

Figure 7 shows the relative effects of the different factors on the mean distance $(\bar{d})$ between the lure and the zebrafish. It allows to obtain a first understanding about the most influential factors on the response. The robot movement $\left(a_{3}\right)$ and the interaction terms between the mock-up shape and the robot movements $\left(a_{13}\right)$ seem to be the most influential factors. However, the effects are limited: none of the factors has a relative effect above $5 \%$.

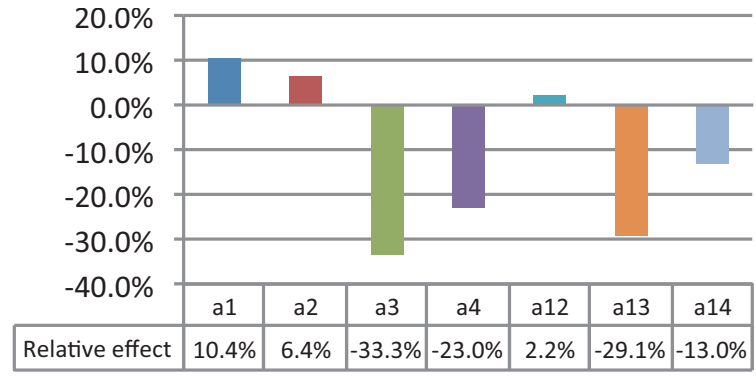

Fig. 8: Relative effects of the factors on the second response, the number of times a zebrafish is situated at less than 10 $\mathrm{cm}$ of the lure $(T)$.

Figure 8 shows the relative effects of the different factors on the number of times $(T)$ a zebrafish is situated at less than $10 \mathrm{~cm}$ of the lure. The same initial conclusions can be drawn than for $\bar{d}$ : the most influential factors on the response are the robot movement $\left(a_{3}\right)$ and the interaction term between the mock-up shape and the robot movement $\left(a_{13}\right)$, followed by the robot's speed $\left(a_{4}\right)$. Furthermore, all coefficients (except $a_{12}$ ) have a relative effect above $5 \%$.

These results are coherent as we can observe that in the case of $\bar{d}$, a small response value indicates a higher acceptation rate of the lure (because of a smaller mean distance), whereas in the case of $T$, a high acceptation rate of the lure is indicated by an elevated response value. As the relative effects of the coefficients were more significant for $T$, we decided to investigate further the results concerning the second response.

Table III presents the ANOVA table of response $T$. In a first step, we decided to include factors with a relative effect below $5 \%$ in the residual (only $a_{12}$ in this case). The ANOVA results show that the three main effects described earlier $\left(a_{3}, a_{13}\right.$ and $\left.a_{4}\right)$ have a probability of being random of $4.2 \%$, $4.8 \%$ and $6.1 \%$ respectively, and can thus be considered as significant effects. The effects of the other factors are not certain because their probabilities of being random are much higher (between 10\% and 20\%). This uncertainty on the other effects is mainly due to the low number of experiments associated with a fractional factorial design (compared to the number of coefficients), which in turn decreases the degrees of liberty left for the residual. One solution would be to reduce the number of coefficients in order to increase the degrees of liberty left for the residual. However, as Table IV shows, including all factors except the three main factors and the constant in the residual does not improve the p-value of the remaining factors. Finally, it was decided to keep all factors with a relative effect above $5 \%$ in the linear model presented in Eq. (1), and thus the following model for the response $T$ was obtained:

$$
Y_{T}=\sum_{i=1}^{4} a_{i} X_{i}+a_{13} X_{13}+a_{14} X_{14}+R e s,
$$

where $Y_{T}$ is the vector of results obtained for the response $T$ and Res the residual. 


\begin{tabular}{lrcrrr}
\hline Effect & SS & df & MS & F & p \\
\hline$a_{0}$ & 14183138 & 1 & 14183138 & 2072.2 & 0.014 \\
$a_{1}$ & 152352 & 1 & 152352 & 22.3 & 0.133 \\
$a_{2}$ & 58825 & 1 & 58825 & 8.6 & 0.209 \\
$a_{3}$ & 1571765 & 1 & 1571765 & 229.6 & 0.042 \\
$a_{4}$ & 749088 & 1 & 749088 & 109.4 & 0.061 \\
$a_{13}$ & 1202801 & 1 & 1202801 & 175.7 & 0.048 \\
$a_{14}$ & 240818 & 1 & 240818 & 35.2 & 0.106 \\
Residual & 6845 & 1 & 6845 & & \\
\hline Total & 18165630 & 8 & & & \\
\hline
\end{tabular}

TABLE III: ANOVA table for response $T$, where factors with relative effects below $5 \%$ are included in the residual.

\begin{tabular}{lrcrrr}
\hline Effect & SS & df & MS & F & p \\
\hline$a_{0}$ & 14183138 & 1 & 14183138 & 123.6 & 0.571 \\
$a_{3}$ & 1571765 & 1 & 1571765 & 13.7 & 0.168 \\
$a_{4}$ & 749088 & 1 & 749088 & 6.5 & 0.237 \\
$a_{13}$ & 1202801 & 1 & 1202801 & 10.5 & 0.191 \\
Residual & 458839 & 4 & 114710 & & \\
\hline Total & 18165630 & 8 & & & \\
\hline
\end{tabular}

TABLE IV: ANOVA table for response $T$, where all factors except the three main factors and the constant are included in the residual.

\section{CONCLUSIONS AND FUTURE WORKS}

In this paper we investigated the acceptance of a robotic fish among a group of real zebrafish while varying several parameters of the experiments, such as the shape of the robot and its motion. The current design of the mobile robot and the global software architecture of our system are also presented with the new powering system of the mobile robots that offers very long multi-robot experiments.

We have noticed that the fish seem to be attracted by the robot, by comparing experiments with and without the lure moving inside the tank. Statistical analyses based on DOE were performed to process the data and build a model. We used a fractional factorial design to reduce the number of experiments to be performed. The reduction of experiments is also a powerful tool when working with real animals, as it will also reduce the amount of required subjects.

Two responses were analyzed: the mean distances between the fish and the robot and the frequency that a zebrafish was at less than $10 \mathrm{~cm}$ from the robot during the experiment. As the relative effects of the coefficients were more significant for the latter, we decided to investigate further the results concerning the second response.

Results show that among the different parameters that were varied during the experiments, coefficients corresponding respectively to the robot's movement, speed and interaction between speed and motion can be considered as being significant, and thus included in the model. As the p-value was not improved by reducing the number of factors, it was decided to keep all the factors in the linear model except the interaction term between the shape of the lure and the robot trajectory.
In further work, we will focus on the design of the lure in order to increase the acceptance of our robot. The trackingbased control will also be improved in order to achieve experiments with higher tangential speeds of the robot.

\section{ACKNOWLEDGMENTS}

This work was supported by the EU-ICT project ASSISIbf, No. 601074. The information provided is the sole responsibility of the authors and does not reflect the European Commission's opinion. The European Commission is not responsible for any use that might be made of data appearing in this publication.

\section{REFERENCES}

[1] J. Halloy, G. Sempo, G. Caprari, C. Rivault, M. Asadpour, F. Tache, I. Said, V. Durier, S. Canonge, J. Ame, C. Detrain, N. Correll,A. Martinoli, F. Mondada, R. Siegwart, and J.-L. Deneubourg, "Social Integration of Robots into Groups of Cockroaches to Control SelfOrganized Choices," Science, vol. 318, no. 5853, pp. 1155-1158, 2007.

[2] J. Krause, A. Winfield, and J.-L. Deneubourg, "Interactive robots in experimental biology," Trends in Ecology and Evolution, vol. 26, no. 7, 2011.

[3] A. Gribovksiy, J. Halloy, J.-L. Deneubourg, and F. Mondada, "The poulbot, a mobile robot for ethological studies on domestic chickens," Symposium on AI-Inspired biology, 2010.

[4] B. Reed and M. Jennings, Guidance on the housing and care of Zebrafish Danio Rerio. Research Animals Department, Science Group, RSPCA, 2010.

[5] N. Abaid, T. Bartolini, S. Macri, and M. Porfiri, "Zebrafish responds differentially to a robotic fish of varying aspect ratio, tail beat frequency, noise and color," Behavioural Brain Research, vol. 233, pp. 545-553, 2012.

[6] M. Aureli, F. Fiorilli, and M. Porfiri, "Portraits of self-organization in fish schools interacting with robots," Physica D: Nonlinear Phenomena, vol. 241, no. 9, pp. 908 - 920, 2012.

[7] J. Faria, J. Dyer, R. Cl'ement, I. Couzin, N. Holt, A. Ward, D. Waters, and J. Krause, "A novel method for investigating the collective behaviour of fish: Introducing "Robofish"," Behavioral Ecology and Sociobiology, vol. 64, pp. 1211-1218, 2010.

[8] D. T. Swain, I. D. Couzin, and N. E. Leonard, "Real-time feedbackcontrolled robotic fish for behavioral experiments with fish schools." Proceedings of the IEEE, vol. 100, no. 1, pp. 150-163, 2012.

[9] T. Landgraf, H. Nguyen, S. Forgo, J. Schneider, J. Schröer, C. Krüger, H. Matzke, R. O. Clément, J. Krause, and R. Rojas, "Interactive robotic fish for the analysis of swarm behavior." in $\operatorname{ICSI}(1)$, ser. Lecture Notes in Computer Science, Y. Tan, Y. Shi, and H. Mo, Eds., vol. 7928. Springer, 2013, pp. 1-10.

[10] F. Bonnet, P. Rétornaz, J. I. Halloy, A. Gribovskiy, and F. Mondada, "Development of a mobile robot to study the collective behavior of zebrafish," in IEEE International Conference on Biomedical Robotics and Biomechatronics, BioRob 2012, 2012.

[11] I. Plaut, "Effects of fin size on swimming performance, swimming behaviour and routine activity of zebrafish danio rerio," Journal of Experimental Biology, vol. 203, pp. 813-820, 2000.

[12] S. Magnenat, P. Rétornaz, M. Bonani, V. Longchamp, and F. Mondada, "Aseba: A modular architecture for event-based control of complex robots," IEEE/ASME Transactions on Mechatronics, vol. 16, no. 2, pp. 321-329, April 2011.

[13] S. Magnenat and F. Mondada, "Aseba meets d-bus: From the depths of a low-level event-based architecture into the middleware realm," IEEE TC-Soft Workshop on Event-based Systems in Robotics (EBS-RO), St. Louis, MO, USA, 2009

[14] D. C. Montgomery, Design and Analysis of Experiments, 7th Edition. International Student Version, 2009.

[15] G. Box, W. Hunter, and J. Hunter, Statistics for Experimenters, An introduction to design, data analysis and model building. Wiley Series in Probability and Mathematical Statistics, John Wyley and Son, NY., 1978.

[16] J.-M. Fürbringer, Design of experiments. EPFL, Doctoral Courses, 2005 . 